\title{
RAPPORT DE SYNTHÈSE : LA FRAGILISATION DE L'ORDRE PUBLIC ÉCONOMIQUE ET LE CONTRÔLE DES ACTEURS PRIVÉS DANS UN ENVIRONNEMENT GLOBALISÉ
}

\section{Benoît Frydman}

De Boeck Supérieur | «Revue internationale de droit économique »

2019/1 t. XXXIII | pages 123 à 130

ISSN 1010-8831

ISBN 9782807393189

DOI 10.3917/ride.331.0123

Article disponible en ligne à l'adresse :

https://www.cairn.info/revue-internationale-de-droiteconomique-2019-1-page-123.htm

Distribution électronique Cairn.info pour De Boeck Supérieur.

(C) De Boeck Supérieur. Tous droits réservés pour tous pays.

La reproduction ou représentation de cet article, notamment par photocopie, n'est autorisée que dans les limites des conditions générales d'utilisation du site ou, le cas échéant, des conditions générales de la licence souscrite par votre établissement. Toute autre reproduction ou représentation, en tout ou partie, sous quelque forme et de quelque manière que ce soit, est interdite sauf accord préalable et écrit de l'éditeur, en dehors des cas prévus par la législation en vigueur en France. Il est précisé que son stockage dans une base de données est également interdit. 


\title{
RAPPORT DE SYNTHÈSE : LA FRAGILISATION DE L'ORDRE PUBLIC ÉCONOMIQUE ET LE CONTRÔLE DES ACTEURS PRIVÉS DANS UN ENVIRONNEMENT GLOBALISÉ
}

\author{
Benoît FRYDMAN ${ }^{1}$
}

Résumé : Ce rapport de synthèse met en évidence les éléments clés de la fragilisation des bases de l'État social et de sa capacité à imposer effectivement aux acteurs privés les contraintes de l'ordre public économique au sens large. Il examine les voies et les limites actuelles de ce contrôle par l'Union européenne, ainsi que les pistes étroites d'un contrôle décentralisé et pluraliste de l'ordre public dans l'environnement global.

Les communications présentées lors de cette journée par les chercheurs du GredegCredeco permettent de donner une certaine idée de la richesse et du dynamisme des recherches et des travaux que développe de manière impressionnante cette équipe de recherche dans les domaines du droit économique. Je suis honoré d'en rapporter ici sur le vif un essai de synthèse. J'ai accepté d'autant plus naturellement que des relations fortes et continues de travail et d'amitié ont été tissées depuis plusieurs années entre l'École de droit économique de Nice et le Centre Perelman de l'Université libre de Bruxelles, non seulement de manière interpersonnelle, mais également collective. Les deux équipes se rencontrent et collaborent régulièrement. Cette collaboration est riche et fructueuse pour nous, car nous nous accordons sur les objets et questions pratiques à traiter, souvent indépendamment des catégories et des concepts doctrinaux classiques, ainsi que sur la manière de les analyser dans leur spécificité technique, éclairée par une approche interdisciplinaire qui permet d'en mesurer les déterminants et les enjeux économiques et sociaux.

Le cadre de ce colloque sur les pouvoirs privés économiques et l'ordre public économique est celui d'une économie mondialisée, dématérialisée et numérisée,

1. Professeur de droit à l'Université libre de Bruxelles, Centre Perelman. 
pour reprendre les termes de Marina Teller dans le domaine financier. Le domaine financier reflète en réalité ce qui est aussi déjà la réalité de nombreux secteurs de l'économie et ce qui le sera bientôt de tous. Nous le voyons avec le succès des plateformes en ligne : la numérisation et la dématérialisation produisent des effets sur le commerce et la distribution autant des biens que des services, y compris sur des activités locales et matérielles comme le transport de personnes ou l'hébergement dans une ville.

Cette révolution technologique et économique modifie les rapports de force qui avaient permis l'établissement et favorisé le développement de l'État social. Dans ce cadre, les États pouvaient imposer un certain nombre de contraintes aux pouvoirs privés économiques en leur prescrivant des règles d'ordre public. Ils avaient également, ainsi que l'Union européenne, la capacité de faire jouer les pouvoirs privés économiques les uns contre les autres dans l'intérêt général, par le moyen du droit de la concurrence, comme l'a souligné Patrice Reis. Enfin, le système de l'État social permettait un prélèvement sur les ressources générées par les acteurs économiques afin de financer les services publics, ainsi qu'un système de protection sociale plus ou moins développé selon les pays.

Or, actuellement, on observe un renversement sur ces trois points fondamentaux. Différentes interventions au cours de la journée ont démontré que les acteurs privés échappent largement aux normes étatiques et tendent à définir leurs propres normes en devenant eux-mêmes les contrôleurs d'un certain nombre d'activités.

Sur le plan de la concurrence, la dynamique s'est également inversée à la faveur de la globalisation qui, en mettant l'investisseur et l'entreprise privée en position de choix de l'État d'accueil ou du système juridique à adopter, crée une concurrence réglementaire entre les États qui provoque une course vers le bas dont les effets délétères, y compris sur les États européens, sont désormais clairement établis ${ }^{2}$. En d'autres termes, la concurrence ne met plus en compétition les acteurs privés les uns contre les autres au bénéfice de l'intérêt général, mais bien les États les uns contre les autres au bénéfice des détenteurs de capitaux et des opérateurs économiques. Cette concurrence opère au détriment de l'intérêt général par la déconstruction progressive des règles et des contraintes imposées aux acteurs privés.

Le troisième point concerne le renversement des flux économiques, comme l'ont souligné certaines interventions. Les prélèvements sur les rendements des capitaux, de même que sur les bénéfices des sociétés, ont considérablement baissé à la fois par l'effet de la concurrence fiscale et sociale et des dispositifs et mécanismes d'évitement et de fraude. On observe aujourd'hui un transfert non plus du capital vers le travail, mais bien du travail vers le capital, non seulement par la

2. Pour une étude comparée de ces effets, voyez B. Frydman, « Mesurer l'impact de la mondialisation sur les droits nationaux. Rapport général sur la mondialisation et sources du droit », in La mondialisation, Journées allemandes de l'Association Capitant, vol. 66, Bruxelles, Larcier, 2017. 
modification de l'arbitrage entre la rémunération des facteurs travail et capital au sein des entreprises, mais également au niveau de la fiscalité, puisque les impôts sur le travail et la consommation servent à rembourser la dette, c'est-à-dire à rémunérer les détenteurs de capitaux. Ainsi, comme l'a bien montré Caroline LequesneRoth concernant les dettes souveraines, il s'agit de prélever des ressources sur les États qui les prélèvent eux-mêmes sur le travail tout en s'engageant dans la réduction des dépenses publiques et donc des services publics pour assurer la charge de la dette.

Face à ce triple constat, on peut se demander si les pouvoirs publics sont encore en mesure d'imposer le respect d'un ordre public économique aux pouvoirs et aux intérêts privés. D'autant que, comme l'ont indiqué Patrice Reis et Jean-Sylvestre Bergé au cours des débats, la frontière entre le public et le privé, qui définissait non seulement la summa divisio du droit, mais le fondement de la philosophie politique libérale, devient de plus en plus floue et poreuse. Nous observons non seulement que des fonctions de régulation et de contrôle sont confiées à des organismes qui sont en réalité aux mains du secteur privé, mais également à la capture par les intérêts privés d'agences et d'institutions publiques qui sont ainsi mises à leur service.

Cette infiltration du privé est très préoccupante par rapport à la mission de ces organismes qui n'ont pas seulement pour fonction d'assurer le bon fonctionnement du marché, par le moyen de la concurrence, mais plus largement de sauvegarder l'ordre public économique. Selon Gérard Farjat, auquel se réfèrent plusieurs intervenants, l'ordre public économique ne se limite pas aux interventions qui assurent ou rétablissent le bon fonctionnement des marchés, mais comprend aussi d'autres formes d'interventions pour limiter les intérêts privés dans les buts d'intérêt général que sont la protection sociale, la protection de l'environnement, la sécurité et les droits de l'homme.

Quant au droit de la concurrence lui-même, l'exposé de Fréderic Marty nous montre comment cet outil, mis au point d'abord aux États-Unis dans les premières décennies du $\mathrm{XX}^{\mathrm{e}}$ siècle, a été édenté dans ce pays par la conception néo-classique de l'économie, qui s'est par ailleurs imposée par le consensus de Washington dans les instances internationales. Selon la théorie néo-classique, qui repose sur le mythe du marché autorégulateur et plaide donc pour un minimum d'interventions publiques, les monopoles eux-mêmes ne doivent pas être condamnés ou combattus $^{3}$. Un monopole peut très bien jouer dans l'intérêt du consommateur et favoriser les innovations technologiques. De toute façon, l'économie de marché y remédie par elle-même puisque les pratiques abusives du monopoliste vont susciter et favoriser l'émergence de concurrents.

3. Rappelons que c'est au nom de la même théorie que les autorités de surveillance des marchés avaient laissé faire, et dans une large mesure laissent encore faire, des pratiques d'ingénierie financière qui ont précipité en 2007 et 2008 le déclenchement de la crise financière et économique mondiale. 
Cette thèse a notamment servi à justifier l'apathie complète des autorités de la concurrence à l'égard des GAFAM, géants économiques, produits de la révolution technologique numérique, que sont Google, Amazon, Facebook, Apple et Microsoft, dont plusieurs se trouvent pourtant en situation de position dominante. Ainsi, au cours du mois d'avril 2018, lors de l'audition de Mark Zuckerberg devant le Sénat américain, celui-ci s'est trouvé complètement démuni pour répondre à un sénateur qui lui demandait de désigner son ou ses concurrents.

Facebook ou Google se sont effectivement constitués en monopoles naturels, à l'image des réseaux physiques de distribution électrique ou des réseaux de chemins de fer. La masse de données et d'utilisateurs dont ils bénéficient les rend particulièrement efficaces en même temps qu'elle fait obstacle à l'entrée d'un nouveau concurrent. Les opposants à toute intervention font valoir que cette situation de domination est précaire dès lors que ces entreprises sont à la merci d'une nouvelle innovation technologique ou commerciale. Ainsi, on constate que le réseau Facebook stagne, alors qu'Instagram progresse, avec plus d'un milliard d'utilisateurs en 2018 (contre plus de trois pour Facebook). C'est exact, mais sans compter le fait que Facebook a racheté Instagram, qui se trouve désormais sous son contrôle. De manière générale, ces acteurs ultra-dominants rachètent un très grand nombre d'innovations et d'entreprises prometteuses, en recourant le cas échéant à des pressions pour leur faire obstacle ou les éliminer du marché en cas de refus.

Or ces entreprises dominantes se procurent une part non négligeable de leurs bénéfices par des activités très contestables sur le plan de leur licéité et dangereuses pour l'ordre public démocratique et l'État de droit. Ainsi, l'audition de Marc Zuckerberg devant le Sénat américain était motivée par l'affaire Cambridge Analytica. Cette affaire a révélé aux autorités et au grand public comment Facebook avait donné accès contre rémunération à des masses de données personnelles de ses abonnés. Les données cédées à Cambridge Analytica ont ensuite été utilisées pour influencer à leur insu leur vote, notamment pour l'élection présidentielle américaine de 2016. Beaucoup plus largement, le «modèle économique » reposant sur l'espionnage des personnes connectées au réseau par leur téléphone, leur ordinateur, d'autres appareils ou des objets, la collecte par ce moyen d'informations les concernant et leur profilage, la cession de ces données à des tiers, de même que leur exploitation à des fins commerciales et politiques, pose désormais un problème majeur d'ordre public.

En dépit de ces graves questions, les autorités américaines s'abstiennent pour l'instant de toute intervention juridique contraignante à l'égard de ces géants du Net qui sont à la fois des entreprises américaines et des super-champions globaux. La situation est différente pour les autorités de la concurrence européennes qui ont, à plusieurs reprises, infligé de lourdes amendes notamment à Microsoft et à Google pour violation du droit européen de la concurrence. Il est nécessaire que de telles actions s'intensifient sans se laisser abuser par la théorie économique des bénéfices du monopole naturel. Il serait au demeurant complètement paradoxal qu'alors 
que l'Union européenne s'est attachée à détruire certains monopoles naturels dans les industries de réseaux classiques des télécommunications, de l'électricité et des chemins de fer nationaux, elle s'abstienne d'agir à l'égard des réseaux nouveaux en dépit de leur puissance économique gigantesque et des abus et dangers considérables que leurs activités suscitent. Dans l'état actuel des choses, le droit de la concurrence constitue probablement le dispositif le plus puissant dont nous disposons pour tenir en respect ces acteurs économiques privés en Europe.

L'intervention de Mehdi Mezaguer a mis en lumière que la Commission européenne utilise également certaines armes du droit de la concurrence, sans se limiter à un secteur particulier, contre certaines actions imputables aux États membres eux-mêmes. Nous avons vu que les États, censés faire eux-mêmes la police de la concurrence, tendent, sous la pression de la concurrence normative, à aider certaines entreprises à se soustraire à leurs propres règles nationales, dans l'espoir de les attirer ou de les maintenir chez eux. Tel est spécialement le cas en matière fiscale, notamment par la pratique des rescrits fiscaux ou tax ruling. L'action de la Commission, qui combat ces pratiques, en les qualifiant d'aides d'État illégales, et oblige les entreprises à rembourser les avantages dont elles ont bénéficié indûment aux États, suscite tout particulièrement l'attention et l'intérêt. Il s'agit d'une question vitale puisque la fuite et l'abandon des ressources fiscales signent le déclin irrémédiable de l'autorité publique et de sa capacité à assurer ses missions de service public.

Mais aussi ingénieuse qu'elle apparaisse, l'application à l'administration fiscale de l'arme juridique de la lutte contre les aides d'État n'est qu'un pis-aller qui, à défaut d'une certaine harmonisation fiscale européenne, ne pourra entraver les effets destructeurs de la concurrence fiscale intra-européenne. Il faut bien constater que l'Union européenne, à l'instar des États membres, joue en l'occurrence double jeu. Si elle lutte ainsi contre les effets délétères de la concurrence interétatique en matière fiscale, elle a elle-même largement contribué à institutionnaliser et à intensifier cette concurrence intra-européenne entre les États membres. Depuis l'adoption de la « nouvelle approche » par la Commission Delors pour réaliser le marché intérieur, elle a substitué, à une politique d'harmonisation législative, une politique qui, par la reconnaissance de l'équivalence mutuelle des législations et le libre choix des acteurs économiques quant à leur point d'ancrage national sur un marché européen sans barrières, conduit de manière nécessaire à la mise en concurrence des réglementations étatiques, en donnant le plus souvent une prime au moins-disant normatif.

Nous sommes ainsi passés d'une harmonisation par les règles à une harmonisation par la concurrence. Madame Orianne Thibout a mentionné cette règle française, qui a pour origine une directive européenne, sur la désignation d'un code de conduite de gouvernance d'entreprise. Cette règle a pour objet de faire jouer la concurrence normative en droit des sociétés. Les sociétés se constituent dans les ordres juridiques étatiques, dont le droit des sociétés, mais aussi le droit social 
et le droit fiscal, ainsi que le droit de l'environnement, leur apparaissent les plus favorables au sein de l'Union. Cette concurrence des droits nationaux en droit des sociétés atteint son summum lorsque la Cour de justice de l'Union européenne permet à une société établie dans un État d'adopter une forme juridique organisée par un autre ordre juridique. Il en va de même en matière de droit social par le biais de la pratique des travailleurs détachés. Dans ces conditions, il apparaît difficile de récupérer par le moyen du droit de la concurrence les pans d'un ordre public économique que l'on a préalablement fragilisé en jouant à la baisse la concurrence juridique intra-européenne.

La question se pose dès lors de savoir comment le droit peut retrouver prise sur les acteurs privés dans le contexte qui vient d'être décrit pour faire valoir des buts d'intérêt général. Rejetant, comme falsifiée empiriquement, la doctrine libérale classique selon laquelle l'intérêt public serait une propriété émergente des interactions spontanées des intérêts privés, l'idée d'un État ou d'une autorité publique mondiale, qui garantirait un ordre public économique mondial, apparaît également complètement irréaliste et probablement pernicieuse. Nous sommes dès lors conduits à rechercher s'il est possible de construire une forme d'ordre public économique global décentralisé et forcément pluraliste.

Le premier élément, et il est fondamental, est que les États y ont un rôle essentiel à jouer. Cependant, pour que leur action gagne en efficacité, il est nécessaire d'ajuster les modalités de leurs actions aux réalités économiques qu'il leur faut regarder en face. Il convient à cet égard de renoncer à certaines habitudes héritées de la souveraineté où il suffisait, du moins le croyait-on, de décréter pour être obéi. La France s'est rendu compte depuis plusieurs années que l'on peut réguler sans être souverain, à la condition d'agir de manière intelligente, et même rusée, ainsi que l'a souligné Orianne Thibout ${ }^{4}$. La loi sur les nouvelles régulations économiques du 15 mai 2001, qui impose le reporting non financier aux sociétés cotées, en constitue un bon exemple et sert de modèle pour l'Union européenne. Plus récemment la loi du 25 mars 2017 sur le devoir de vigilance, sur laquelle a porté l'intervention de Larios Mavoungou, nous en donne une nouvelle manifestation.

Ces deux législations s'inscrivent dans la logique d'une responsabilisation sociétale des entreprises. L'intervention de l'État confère à celle-ci une certaine effectivité, y compris en donnant à des tiers les moyens juridiques de contrôler les activités des entreprises et d'engager le cas échéant leur responsabilité juridique. Elles ont en commun d'étendre leurs effets bien au-delà des limites du territoire national. Elles s'inscrivent par là aussi dans une logique de régulation par les flux, que préconise Jean-Sylvestre Bergé. Il s’agit, pour les États notamment, par le

4. Sur la nécessité de la ruse dans la lutte globale pour le droit, le lecteur intéressé peut consulter mon Petit manuel pratique de droit global, coll. L'Académie en poche, Bruxelles, Académie royale de Belgique, 2014. 
biais de la RSE, de tenter d'imposer des préoccupations d'ordre public sur toute la longueur de la chaîne de valeur.

Au cours de cette journée, plusieurs interventions ont mis en avant l'idée que l'on a autant de responsabilité que de pouvoir, principe qui n'a pas encore reçu une pleine reconnaissance juridique, mais qui pourrait constituer à l'avenir un principe général dans le rapport entre ordre public économique et pouvoirs privés économiques. Ce principe sous-tend d'ailleurs le concept de la responsabilité sociétale des entreprises. La norme ISO 26000 sur la responsabilité sociale des organisations l'exprime d'ailleurs par la formule selon laquelle la responsabilité d'une organisation s'étend à l'ensemble de sa « sphère d'influence ». Dans le même esprit, Larios Mavoungou a affirmé, en ce qui concerne la loi sur le devoir de vigilance, que la société mère était responsable non seulement à l'égard de ses filiales directes et indirectes, mais aussi en amont et en aval de la chaîne de valeur à condition qu'il y ait des relations commerciales établies.

Il faut enfin souligner le rôle du juge dans la garantie de l'ordre public économique. Ce juge prendra souvent, dans l'environnement mondialisé, la figure de l'arbitre. Dans leur intervention, Jean-Baptiste Racine et Loïs Dossios ont montré que l'ordre public économique au niveau international n'est pas édicté par une institution législatrice, mais bien une construction des juges et notamment des arbitres. C'est l'illustration la plus frappante d'un ordre public décentralisé et pluraliste. Comme l'a montré Jean-Baptiste Racine, les nécessités de l'ordre public sont la principale limite que les arbitres d'un litige international peuvent opposer à l'intérêt bien compris des parties. L'étude de la pratique permet de constater l'apparition dans les arguments des parties d'une sorte d'exception d'ordre public qui commence à être prise en compte par les arbitres. On voit ainsi des acteurs privés, désignés par d'autres acteurs privés, pour une mission de justice privée, qui pourtant reconnaissent l'existence d'un ordre public économique.

En conclusion, il est clair que la globalisation économique et la révolution numérique affaiblissent considérablement les États dans leurs bases et leur capacité à imposer les contraintes de l'ordre public et de l'intérêt général aux acteurs économiques privés transnationaux. Cet affaiblissement suscite une crise politique et sociale majeure au sein des États. L'Union européenne ne fournit pas une alternative dès lors qu'elle se limite à une conception étroite de l'ordre public économique, centrée sur le droit de la concurrence, sans réussir à intégrer véritablement d'autres dimensions essentielles, en particulier sociale et fiscale. Dans ces conditions difficiles, où le rapport des forces entre l'autorité publique et les intérêts privés favorise nettement ces derniers, le contrôle juridique d'acteurs économiques privés globalisés semble passer pour l'instant par les voies étroites d'une législation rusée qui s'étendrait aux flux économiques par-delà les frontières et l'intervention du juge ou de l'arbitre comme garants d'un ordre public international. Mais ces voies ne sont clairement pas suffisantes. 


\section{SUMMARY REPORT: THE WEAKENING OF THE ECONOMIC PUBLIC ORDER AND THE REGULATION OF PRIVATE ACTORS IN A GLOBALIZED ENVIRONMENT}

This summary report highlights the key elements explaining the weakening of the foundations of the welfare state and of its means to effectively impose on private actors the constraints of the economic public order in the broadest sense. It also examines the approaches and current limits of the European Union in this regulation, as well as the narrow path of a decentralized and pluralistic regulation of public order in the global environment.

Mots clés : droit global, ordre public économique, pouvoirs publics, rapports de pouvoir, pouvoirs privés économiques

Keywords: global law, economic public order, public powers, power relations, private economic powers 